\title{
Test Model and Coverage Analysis for Location-based Mobile Services
}

\author{
Tao Zhang \\ School of software and \\ Microelectronic \\ Northwest Polytechnical University \\ Xi'an, China \\ tao_zhang@nwpu.edu.cn
}

\author{
Jerry Gao \\ Department of Computer \\ Engineering \\ San Jose State University \\ San Jose, USA \\ jerry.gao@sjsu.edu
}

\author{
Oum-EI-Kheir Aktouf \\ LCIS Laboratory \\ University of Grenoble, \\ France \\ Oum-El-Kheir.Aktouf \\ @)grenoble-inp.fr
}

\author{
Tadahiro Uehara \\ Software Innovation \\ Laboratories \\ Fujitsu Laboratories LTD. \\ Japan \\ uehara.tadahiro@jp.fujitsu.com
}

\begin{abstract}
Location-based services (LBS) are very important mobile app services, which provide diverse mobility services for mobile users anywhere and anytime. This brings new demands, issues, and challenges in mobile application testing. Today, mobile applications provide location-based service functions based on dynamic location contexts, mobile users and their travel patterns to deliver location-based mobile data, and service actions. Current software testing methods do not consider location-based validation coverage. Hence, there is a lack of research results addressing location-based mobile application testing. This paper focuses on mobile LBS testing. A novel test object model is proposed for quality validation of location-based mobile information services. In addition, the related test coverage metrics are also presented. These metrics can be useful for test engineers in designing test cases. A case study based on student testers is reported to demonstrate the potential application of the proposed model.
\end{abstract}

Keywords-component; Location-based service; mobile app; mobile testing; test model; test coverage; test metrics

\section{INTRODUCTION}

In recent years, more and more diverse mobile applications (mobile apps) have been developed to support different applications in social, news, tourism, health, business, and other domains. Hundreds of new apps are released daily, e.g. about 300 new apps appear on Apple's App Store each day. In turn, 750 million Android and iOS apps are downloaded each week [1].

Mobile Location-based services are services enhanced with positional data, which are provided by mobile apps using GPS, digit maps, and other techniques [2]. Many mobile apps provide interesting and convenient location-based services and functions. The mobile app Yelp (www.yelp.com) recommends nearby shops, restaurants, etc. In the social network mobile app Loopt (www.loopt.com), the users receive notifications whenever their friends are nearby. The mobile app Waze (www.waze. com) reports nearby traffic jams, policemen, gas stations and friends.
Mobile LBS have been identified as the one of most important features of mobile app services [3], and have become one hot research topic in mobile computing domains. In the recent years, there have been a number of published research papers addressing LBS. However, most papers primarily focus on the following areas:

- LBS infrastructure, framework, and architecture [4][5][6][7];

- $\quad$ Privacy preserving for LBS [8][9][10];

- Positioning techniques for LBS [11][12];

- $\quad$ LBS databases [13][14].

Till now, there are a few publications discussing mobile LBS testing. There is a lack of systematic and effective test models and methods for test engineers to address LBS testing. The test engineers often ask mainly the following questions about LBS testing:

1) How to select and test a set of locations for LBS?

2) How to test different types of location objects?

3) How to test millions instances of location objects and their information?

4) How to test moving objects with dynamical moving paths and patterns?

This paper focuses on cost-effective mobile LBS testing issues. The major contributions of this paper include four areas: 1) discussing the basic concepts, scope and challenges of mobile LBS testing; 2) proposing a novel test object model for mobile LBS testing; 3) some coverage metrics are defined for evaluating quality of mobile LBS testing; and 4) providing case studies to show the effectiveness of the proposed test model.

The rest of the paper is structured as follows. Section II discusses basic concepts and main challenges of LBS testing. Section III presents a novel test model and a test approach for location-based mobile information services. Section IV reports 
the results of a case study. Section $\mathrm{V}$ reviews and discusses published papers and related research work. Finally, concluding remarks and future research directions are given in Section VI.

\section{UNDERSTANDING MoBILE LBS TESTING}

\section{A. What is Mobile LBS Testing?}

Location-based services are information, entertainment, services that are conveniently accessible by mobile users through GPS-enabled portable devices and mobile networks (e.g., 2G/3G/4G cellular telephones and Wi-Fi networks). Some organizations and scholars have defined LBS, and the classic definition is presented below.

Definition 1: LBSs are information services accessible with mobile devices through the mobile network and utilizing the ability to make use of the location of the mobile device [15].

Based on our recent literature survey, there is a lack of published papers on LBS testing for mobile apps, and also no precise definition of mobile LBS testing. Here we define it as below.

Definition 2: Mobile LBS testing refers to testing activities for native and Web apps on mobile devices to ensure quality in location-based functions, behaviors, information, and quality of service.

\section{B. The Classifications of Mobile LBS}

In recent years, mobile LBS have become more and more popular, and many mobile LBS apps are developed in various domains, such as navigation, information, emergency, advertising, tracking, games, management, and leisure. According to behavior characteristics of LBS, mobile LBS are divided into three types: basic location services, location context information services, and location context interactive services. Those services have different characters and features, and are compared in Table I.

TABLE I. MOBILE LBS SERVICES CHARACTERS

\begin{tabular}{|c|c|c|c|}
\hline Objects & Basic location & $\begin{array}{c}\text { Location context } \\
\text { services } \\
\text { Services }\end{array}$ & $\begin{array}{c}\text { Location context } \\
\text { interactive } \\
\text { services }\end{array}$ \\
\hline Positions & Moving along paths & Fixed positions & $\begin{array}{c}\text { Moving along } \\
\text { different paths }\end{array}$ \\
\hline $\begin{array}{c}\text { Typical } \\
\text { Services }\end{array}$ & $\begin{array}{c}\text { Map services, } \\
\text { Location services, } \\
\text { Navigation services }\end{array}$ & $\begin{array}{c}\text { Search services, } \\
\text { Access services, } \\
\text { Update services }\end{array}$ & $\begin{array}{c}\text { Interactive } \\
\text { services }\end{array}$ \\
\hline $\begin{array}{c}\text { Service } \\
\text { mode }\end{array}$ & Push way & $\begin{array}{c}\text { Push way, } \\
\text { Pull way }\end{array}$ & $\begin{array}{c}\text { Push way, } \\
\text { Pull way }\end{array}$ \\
\hline $\begin{array}{c}\text { Real-time } \\
\text { feature }\end{array}$ & High & Low & High \\
\hline $\begin{array}{c}\text { Infor- } \\
\text { mation }\end{array}$ & $\begin{array}{c}\text { Digital map } \\
\text { information }\end{array}$ & $\begin{array}{c}\text { Location context } \\
\text { information }\end{array}$ & $\begin{array}{c}\text { Mobile Object } \\
\text { properties }\end{array}$ \\
\hline & $\begin{array}{c}\text { Map } \\
\text { Navigation } \\
\text { Emergency }\end{array}$ & $\begin{array}{c}\text { Advertising } \\
\text { Tracking } \\
\text { Managements } \\
\text { Leisure }\end{array}$ & $\begin{array}{c}\text { Game, Social } \\
\text { network, } \\
\text { Intelligent } \\
\text { automobile }\end{array}$ \\
\hline Domain & Intormation & \\
\hline
\end{tabular}

Basic location services - The mobile apps provide basic digital maps, location, and navigation services to help mobile users find correct ways to their destination. Some early map and navigation apps provide basic location services for mobile users.

Location context information services - The mobile users search nearby location objects and then access or update information of location objects. Those location objects have fixed positions, and provide static or dynamic information. Mobile users access information with push or pull ways. Most of information services are not real time services. The location context information services are widely used in many domains, for example, business, advertising, management, and leisure.

Location context interactive services - The multiple mobile objects interact with each other in same location contexts. Those mobile objects may be mobile users, smart cars, sensors, or other objects with positions. Location context interactive services provide different behaviors or functions according to locations or location relations between objects. Most of location context interactive services are real-time services, and are applied in some new domains, such as social networks, games and intelligent automobile.

Today, there are hundreds of mobile apps with locationbased information services, such as, Yelp, Booking, etc. So this paper focuses on testing location-based mobile information services.

\section{Why is Mobile LBS Testing Important?}

Mobile LBS have been identified as killer services of mobile apps, which provide many conveniences and interests for mobile users. However, few published papers address the importance of mobile LBS testing. Here, its primary reasons and importance are listed as follows.

Reason \#1: Multiple techniques for LBS - Mobile LBS normally use many different techniques, such as mobile computing, geographic information systems, GPS, cloud computing, Internet, etc. Those techniques make mobile LBS testing more complex.

Reason \#2: Location impact for LBS - Location is the most critical factor for mobile LBS, which affects information, behaviors, functions, performance, dependability, privacy, etc.

Reason \#3: Higher costs of LBS testing - Due to numerous locations, different kinds of location objects with changing information, and moving objects with unpredictable paths, the test engineers have to spend a lot of time and effort on mobile LBS testing.

\section{Test activities and goals}

Mobile LBS testing tends to focus on the following activities and goals:

LBS functionality and behavior testing - activities that validate location-based service functions, such as map and navigation services, information services, interactive services, etc. 
LBS information and data testing - activities that validate LBS information and data, such as, positions, information, moving paths of mobile objects.

LBS QoS testing - activities that evaluate system load, performance, reliability, availability, scalability, and throughput in different location contexts.

LBS security and privacy testing - activities that check user data security and location information privacy.

LBS compatibility and connectivity testing - activities that assess mobile browser and platform compatibility, especially compatibility with different location techniques, and diverse wireless network connectivity.

LBS regression testing - Mobile LBS are updated frequently for fixing faults, adapting to new devices and platform versions, and enhancing functions. Regression testing is continuous for mobile LBS.

\section{E. Scope of mobile LBS testing}

LBS testing focuses on testing and validating location related functions and features of mobile apps. As shown in Figure 1, the scope of LBS testing includes the following six types of testing activities.

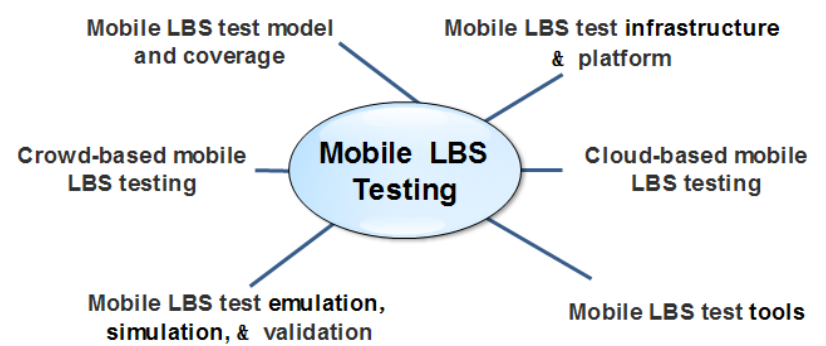

Figure 1. The Scope of Mobile LBS Testing

LBS test model and coverage - This refers to the activities that analyze and model main factors of mobile LBS, such as locations context, location object, location-related function and behavior, etc. The test models are used to generate test cases, and to analyze test coverage.

LBS test infrastructure and platform - This refers to the study of solutions on how to build the infrastructure and platform supporting automatic mobile LBS testing.

Crowd-based mobile LBS testing - This refers to the crowd-based test approach for mobile LBS, which allows to test mobile LBS in more extensive ranges.

Cloud-based mobile LBS testing - This refers to the cloudbased test approach for mobile LBS, which provides dynamic test resources and location simulation for mobile LBS testing.

LBS test emulation, simulation, and validation - This refers to study and apply different mobile LBS testing approaches.

Mobile LBS test tools - This refers to develop test tools to support automatic testing of mobile LBS.

\section{THE TEST MODEL AND COVERAGE}

This section presents a novel test model for location-based mobile information services. Based on this test model, a test approach and some coverage metrics are proposed.

\section{A. The Mobile LBS Test Model}

\section{1) Basic concepts and definitions}

First, some basic concepts about location contexts are defined. These concepts are important to design test cases for mobile LBS.

Definition 3: A location $1_{i}=\left(\operatorname{long}_{i}\right.$, lat $\left.t_{i}\right)$ is a pair of real numbers representing the longitude long $_{i}$ and the latitude ${ }^{l a t_{i}}$ of the location on the Earth surface.

Definition 4: The range range $_{i}\left(l_{i}, r_{i}\right)$ denotes one circular range, the center of the circular range is $l_{i}$, and the radius of the circular range is $r_{i}$.

Definition 5: A path $\operatorname{path}_{i}\left(1_{i 1}, 1_{i 2}, \cdots, 1_{i k}\right)$ denotes a moving path through a set of location points $\left(I_{1}, I_{2}, \cdots, I_{k}\right)$.

\section{2) Test object model}

The test object model for mobile LBS is proposed to represent the core objects and their relations. This model is used to design test cases and help test coverage analysis for mobile LBS. As shown in Figure 2, mobile LBS have four types of core objects: location context, Map, location object and location service.

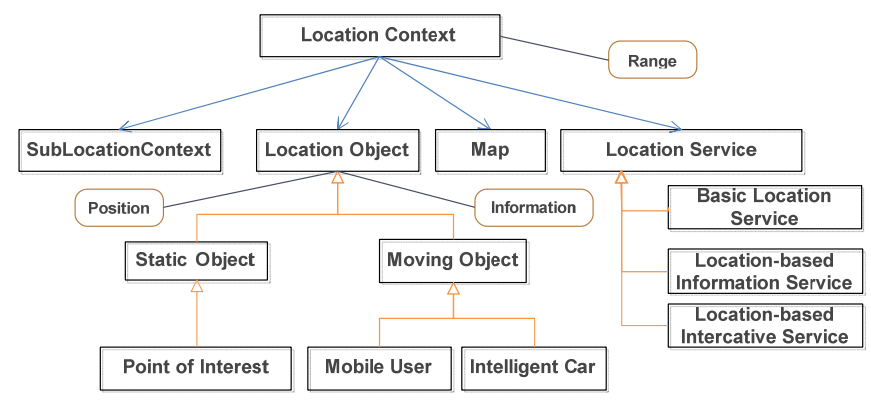

Figure 2. Test object model for mobile LBS

The location context LC for location-based mobile services is denoted as a 4-tulpe

$$
\text { LC }\left(\begin{array}{l}
\text { SubLocationContexts, Map, } \\
\text { LocationObjects, LocationServices }
\end{array}\right) \text {. }
$$

Location context defines the range of mobile LBS, which includes map, a set of sub-location contexts, a set of location objects, and a set of location services.

Sub-location context is part of the location context, and represents a sub area. The location context contains many sub- 
location contexts, and one sub-location context can contains some smaller sub-location contexts.

Map is used to display location contexts, and positions of location objects and mobile users. Map also has some basic behaviors, such as zooming, moving, etc.

Location Objects are a set of objects with positions. There are two types of location objects: static objects and mobile objects. Static objects have fixed positions, such as point of interest. Mobile objects have various moving patterns and paths. Location objects can provide static or dynamic information.

Location Services are a set of services in a location context. The main location context services include basic location services, location-based information services and locationbased interactive services. Those location context services may have various behaviors in different location contexts.

\section{B. The Test Coverage Metrics}

In order to analyze and ensure test quality, some test coverage metrics are defined to evaluate mobile LBS testing.

Definition 6: The test range $R n g_{t}$ is defined as the sum of all tested sub-location context's ranges. It is formulated as below.

$$
R n g_{t}=\sum_{i=1}^{n} R n g_{i}
$$

Here, $R n g_{i}$ is the range of tested sub-location context $S L C_{i}$.

Definition 7: The range test coverage $\operatorname{Cov}_{\text {reg }}$ is defined as the ratio of the sum of all tested ranges $R n g_{t}$ to all ranges $R n g_{a}$. It is formulated as below.

$$
\operatorname{CoV}_{\text {reg }}=\frac{R n g_{t}}{R n g_{a}}
$$

In most situations, only a few ranges are tested because of restricted test resources. Then how to select and prioritize test locations and ranges is a critical issue for mobile LBS testing.

Definition 8: The location object type test coverage $\mathrm{Cov}_{L O t}$ is defined as the ratio of the number of tested location object types Num $_{T L O t}$ to the number of all location object types Num $_{\text {ALOt }}$. It is formulated as below.

$$
\operatorname{COV}_{L O t}=\frac{\operatorname{Num}_{\text {TLOt }}}{\operatorname{Num}_{\text {ALOt }}}
$$

Location object type test coverage is used to ensure that all types of location objects are tested at least once.

Definition 9: The location object test coverage $C O V_{L O i}$ is defined as the ratio of the number of tested location objects
$N_{U L} m_{T L O i}$ to the number of all location objects $N_{U M} m_{A L O i}$. It is formulated as below.

$$
\operatorname{COV}_{L O i}=\frac{\operatorname{Num}_{T L O i}}{\operatorname{Num}_{A L O i}}
$$

Location object test coverage is used to test and evaluate information and behaviors of all location objects. However, there may be thousands of location objects in the location context, and then we have to select a small part of location objects to test.

Definition 10: The services test coverage $\operatorname{CoV}_{S r V}$ is defined as the ratio of the number of tested services Num $_{T S r V}$ to the number of all services $N_{u m_{A S r}}$. It is formulated as below.

$$
\operatorname{Cov}_{S r V}=\frac{\operatorname{Num}_{T S r V}}{\operatorname{Num}_{A S r V}}
$$

Definition 11: The object services test coverage $C O V_{O S r V}$ is defined as the ratio of the number of tested services for every type of location object $N u m_{\text {TOS V }}$ to the number of all services for every type of location object $N_{U m_{A O S T V}}$. It is formulated as below.

$$
\operatorname{CoV}_{\text {OSrV }}=\frac{\text { Num }_{\text {TOSrV }}}{\text { Num }_{\text {AOSrV }}}
$$

The same services may concern different types of location objects, and then we need test services for every type of location object individually.

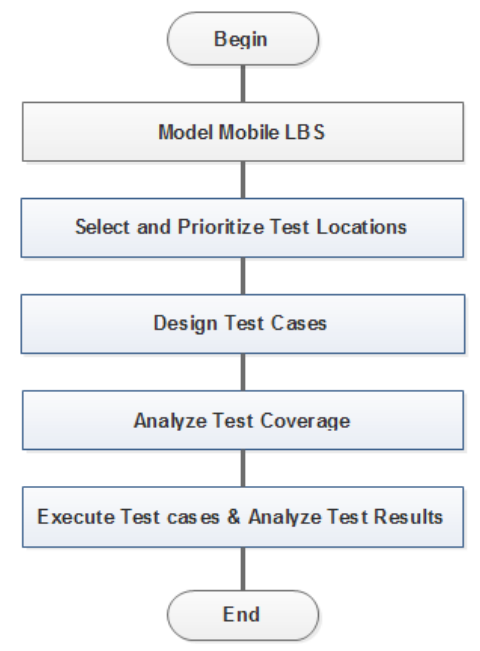

Figure 3. Model-driven test process for mobile LBS

\section{Model-driven Test approach for Mobile LBS}

The model-driven approach that we use for mobile LBS testing consists of five steps. The first step is to model location objects, location context Services for under-test mobile LBS 
using the proposed meta-model. The second step is to select some test locations according to mobile user contexts. The third step is to design test cases according to the test models of mobile LBS. The fourth step is to analyze test coverage of mobile LBS. The last step is to execute test cases and analyze test results. This process is shown in Figure 3.

The main difficulty for mobile LBS testing is to select and prioritize test location contexts and location objects. Simulation-based and emulation-based test approaches are cost-effective to test different location contexts and location objects for mobile LBS. However, field test is necessary for mobile LBS. Then crowd-based testing is a novel and convenient approach to test a large number of location contexts and location objects for mobile LBS.

\section{CASe Study}

To demonstrate the proposed approach for mobile LBS testing, we applied the approach to test one selected mobile app Tripadvisor. We conducted this case study in detail as described below.

\section{A. The Test Model for Tripadvisor}

Tripadvisor provides location-based travel services, which helps travelers to search and book hotels, flights, restaurants based on their locations. The test model for Tripadvisor is presented in Figure 4.

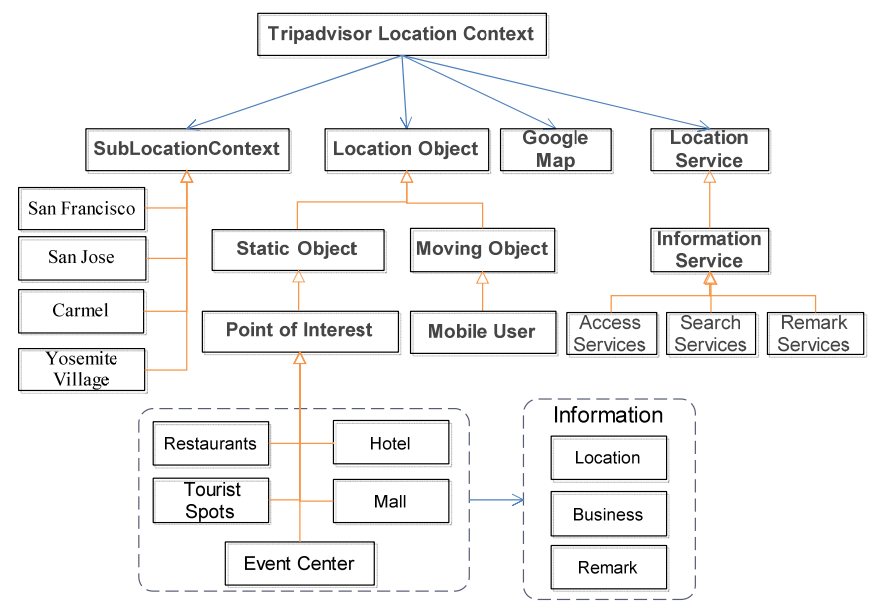

Figure 4. Test Object Model for Tripadvisor

TABLE II. TRIPADVISOR LBS DESCRIPTIONS

\begin{tabular}{|c|c|l|}
\hline \multirow{2}{*}{$\begin{array}{c}\text { Location } \\
\text { context } \\
\text { Services }\end{array}$} & Search Services & $\begin{array}{l}\text { Search nearby location objects, such } \\
\text { as: hotels, restaurants, tourist spots, } \\
\text { malls, and event centers. }\end{array}$ \\
\cline { 2 - 3 } & Access Services & $\begin{array}{l}\text { Access information of nearby location } \\
\text { objects, such as positions, phone } \\
\text { numbers, business hours, and remarks. }\end{array}$ \\
\cline { 2 - 3 } & Remark Services & $\begin{array}{l}\text { Mobile users remark some nearby } \\
\text { location objects by their experiences. }\end{array}$ \\
\hline
\end{tabular}

Tripadvisor provides some location objects, including restaurants, hotels, tourist spots, malls, and event centers. Those location objects have positions, remarks, and business information. Tripadvisor provides some services for mobile users to search, access, and remark location objects. Those services are described in Table II.

\section{B. Test approach for Tripadvisor}

\section{1) Selecting and specifying test locations}

For testing LBS, we must select and specify test positions and ranges firstly. Tripadvisor has been used in many countries and cities, we only test Tripadvisor in San Francisco Bay Area because of restricted test resources. Four different locations are selected, which represent different kinds of location contexts. The details about selected location contexts are described in Table III.

TABLE III. LOCATION CONTEXTS FOR TRIPADVISOR

\begin{tabular}{|c|c|c|c|c|}
\hline LC id & LC1 & LC2 & LC3 & LC4 \\
\hline Location & $\begin{array}{c}\text { San Francisco } \\
\text { City }\end{array}$ & $\begin{array}{c}\text { San Jose } \\
\text { City }\end{array}$ & Carmel & $\begin{array}{c}\text { Yosemite } \\
\text { Village }\end{array}$ \\
\hline Description & Big city & $\begin{array}{c}\text { Medium } \\
\text { city }\end{array}$ & Small city & $\begin{array}{c}\text { Tourism } \\
\text { area }\end{array}$ \\
\hline Position & 37.805623 & 37.808517, & 36.554986 & 37.742004, \\
-122.406722 & -122.411934 & -121.922041 & -119.582939 \\
\hline Range & $600.6 \mathrm{~km}^{2}$ & $466.1 \mathrm{~km}^{2}$ & $2.798 \mathrm{~km}^{2}$ & $3081 \mathrm{~km}^{2}$ \\
\hline Restaurants & 5097 & 1953 & 150 & 28 \\
\hline Hotels & 233 & 73 & 23 & 30 \\
\hline Tourist Spots & 290 & 62 & 18 & 57 \\
\hline Events & 419 & 46 & 36 & 14 \\
\hline Malls & 247 & 20 & 65 & 1 \\
\hline Sum & $\mathbf{6 2 8 9}$ & $\mathbf{2 1 5 4}$ & $\mathbf{2 9 2}$ & $\mathbf{1 3 0}$ \\
\hline
\end{tabular}

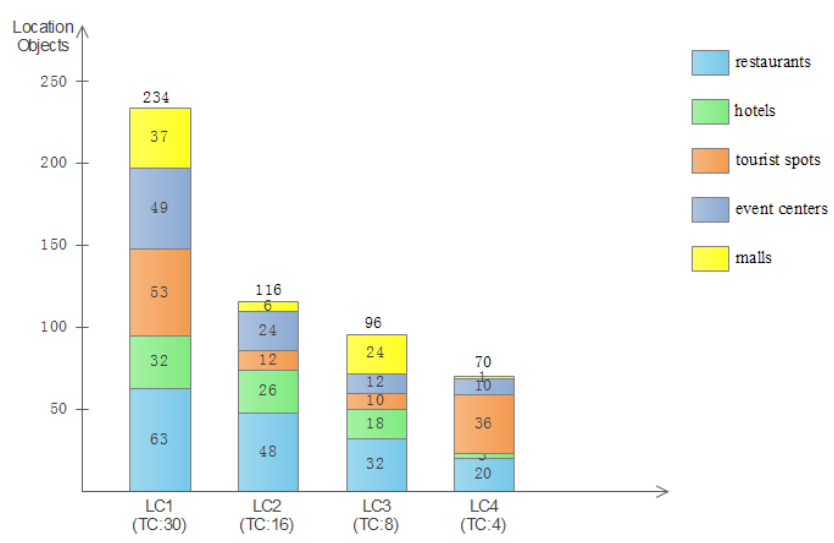

Figure 5. Tripadvisor test cases and tested location objects

\section{2) Designing test cases for Tripadvisor}

Based on location contexts, test cases (TC) are designed for mobile LBS testing. All types of location objects and location context services should be tested at least once. We designed 58 test cases for Tripadvisor LBS in 4 location contexts based on the proposed test model. The test cases and tested location objects are shown in Figure 5.

As shown in Figure 6, we design one test case for Tripadvisor to test searching nearby hotels in San Jose. Then the 29 hotel objects are shown in the map. 


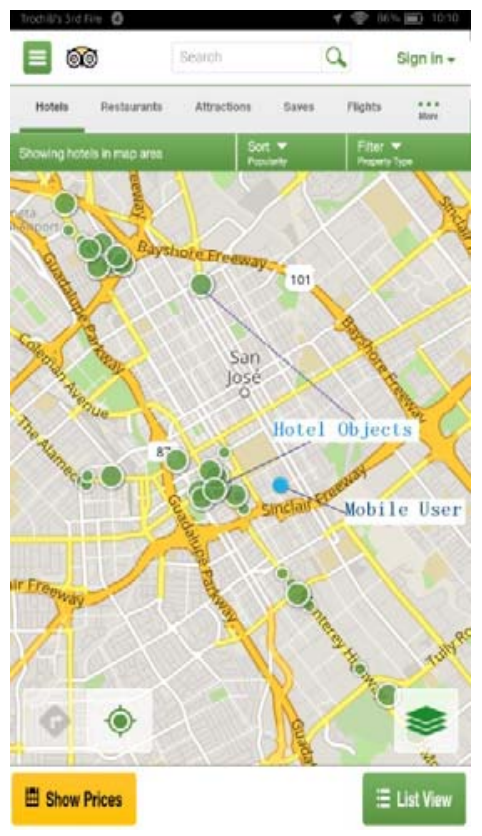

Figure 6. A test case example

\section{3) Analyzing test results and coverages}

The five types of location objects have been tested, and three location context services have also been tested. We only selected and tested four location contexts, so this is too small to calculate range test coverage. There are millions of location objects in Tripadvisor, we tested 516 location objects from 8862 located objects in the four selected location contexts. Finally, 4 faults have been found. The test coverages are described in Table IV, and a fault about losing mobile user position is shown in Figure 7.

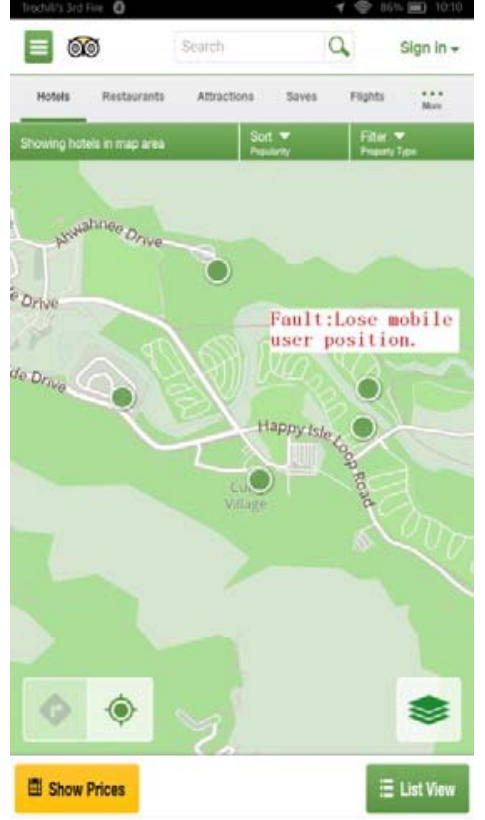

Figure 7. A fault for Tripadvisor
TABLE IV. TRIPADVISOR LBS TEST COVERAGE

\begin{tabular}{|c|c|c|c|c|c|}
\hline $\begin{array}{c}\text { Test } \\
\text { Cases }\end{array}$ & $\operatorname{Cov}_{L O t}$ & $\operatorname{Cov}_{L O i}$ & $\operatorname{Cov}_{S r V}$ & $\operatorname{Cov}_{\text {OSTV }}$ & Faults \\
\hline 58 & $5 / 5$ & $516 / 8862$ & $3 / 3$ & $15 / 15$ & 4 \\
\hline
\end{tabular}

Using our proposed approach, the test engineers design test cases for testing more location objects and services based on the test model. According to table IV, all location objects and services are tested fully. This approach helps to reach high test coverage, to improve test effectiveness and save test costs.

\section{RELATED WORK}

Nowadays, mobile LBS have become one hot research topic in mobile computing domain, and many papers have been published to address different areas in mobile LBS.

Some mobile LBS frameworks and architectures have been proposed. Chengcheng Dai proposes a LBS framework using vehicular ad-hoc networks [4]. R. Gobi proposes a communication framework for data management in LBS [5]. A conceptual framework for personalized location-based tourism apps leveraging semantic web to enhance tourism experience is proposed by Mahmood [6]. Rui Jose proposes the AROUND architecture for supporting location-based services in the Internet environment [7].

The privacy issues are serious for LBS. Ben Niu proposes two dummy-based solutions to achieve k-anonymity for privacy-area aware users in LBSs by considering that side information may be exploited by adversaries [8]. K. G. Shin presents a comprehensive overview of the existing schemes for protecting LBS users' privacy [9]. An adaptive location privacy-preserving the system is presented, which allows a user to control the level of privacy disclosure with different quality of location-based services [10].

Position technique is the key for LBS. HuangChi Chen proposes a novel indoor positioning technique based on neural networks [11]. Al Nabhan presents a new strategy in achieving highly reliable and accurate position solutions fulfilling the requirements of Location-Based Services (LBS) pedestrians' applications [12].

Suprio Ray presents an in-memory database technique for location-based service, and introduces a parallel spatiotemporal index to support historical, past and predictive (future) location-based queries [13][14].

However, there are a few publications about mobile LBS testing. Jerry Gao discusses the issues and difficulties of mobile LBS testing [16]. Ke Zhai proposes a suite of metrics for prioritizing test cases for regression testing of LBS [17]. Huichun Chu proposes a two-tier test approach for locationaware mobile learning systems [18]. Solveig Bjørnestad presents an example study about evaluation of a location-based mobile news reader [19]. Jiang $\mathrm{Yu}$ analyzes test requirements, and presents a scalable testing framework for mobile LBS [20]. However, those papers do not discuss test models for mobile LBS. 
We have proposed an initial test model for function services of mobile LBS [21]. In this paper, we improve and perfect the test model by considering location context, range, location objects, moving pattern and path, and some new metrics are defined for evaluating mobile LBS test coverage.

\section{CONCLUSION AND FUTURE WORK}

Recently, mobile LBS have become popular among research groups. Because mobile LBS provide contextsensitive functions based on location information, this brings many new difficulties and challenges for mobile LBS testing.

This paper analyzes the main factors about mobile LBS testing. A new test object model has been proposed, and some metrics are defined to evaluate test coverage for mobile LBS. The test object model and test metrics help test engineers to design test cases for mobile LBS, and to improve test quality by higher test coverage.

Future research directions include four areas: a) selecting and prioritizing location contexts and location objects for mobile LBS testing; 2) enhancing the test approach to validate and verify correctness and timeliness of location-based services; 3) designing and specifying moving paths for mobile objects; and 4) developing automatic test tool supporting mobile LBS testing with emulation and simulation.

\section{ACKNOWLEDGEMENT}

This research project was supported by National Natural Science Foundation of China (Program No. 61103003). This research project was jointly funded by Fujitsu Research Lab. on Mobile SaaS Testing from 2013-2015.

\section{REFERENCES}

[1] Adrian Holzera, and Jan Ondrusb. "Mobile application market: A developer's perspective", Telematics and Informatics, 2014, 28(1): pp.22-31.

[2] Sergio Ilarri, Arantza lllarramendi, Eduardo Mena, Amit Sheth, "Semantics in Location-Based Services," IEEE Internet Computing, vol. 15(6), 2011, pp.10-14.

[3] Shang-Pin Ma, Wen-Tin Lee, and Chia-Hsu Kuo, "Location Explorer with information services: A mobile application to deliver locationbased web services", 2013 IEEE International Symposium on NextGeneration Electronics (ISNE 2013), pp.283 - 286

[4] Chengcheng Dai, Chiyin Chow, and Jiadong Zhang. "Utilizing road-side infrastructure for location-based services in vehicular ad-hoc networks", the 8 th International ICST Conference on Communications and Networking, 2013, pp.546-551.

[5] R. Gobi, Dr. E. Kirubakaran, and Dr. E. George Dharma Prakash Raj. "ComFrame: A Communication Framework for Data Management in Mobile Location Based Services", International Journal of Computer Science and Telecommunications, vol.3(7), 2012, pp.65-68.

[6] Mahmood, F.M., Bin Abdul Salam, Z.A. "A conceptual framework for personalized location-based Services (LBS) tourism mobile application leveraging semantic web to enhance tourism experience", the 3rd IEEE International Conference on Advance Computing (IACC), 2013, pp.287 $-291$

[7] Mohammad AL Nabhan, Suleiman Almasri, Vanja Garaj, Wamadeva Balachandran, and Ziad Hunaiti, "Client-Server Based LBS Architecture: A Novel Positioning Module for Improved Positioning Performance", International Journal of Handheld Computing Research, vol.1(3), 2010, pp.1-18

[8] Ben Niu, Zhengyan Zhang, Xiaoqing Li, and Hui Li "Privacy-area aware dummy generation algorithms for Location-Based Services ", 2014 IEEE International Conference on Communications (ICC), 2014 pp.957- 962

[9] K. G. Shin, X. Ju, Z. Chen, and X. Hu, "Privacy protection for users of location-based services," IEEE Wireless Communications, vol.19(1), 2012, pp. 30-39.

[10] Zhu, I., K.-H. Kim, and P. Mohapatra. "An Adaptive Privacy-Preserving Scheme for Location Tracking of a Mobile User." in IEEE Internation Conference on Sensing, Communication, and Networking. New Orleans, USA, 2013, no pp. 9.

[11] Chen HuangChi, Chen YuJu, Chen ChihYung, Wang Shuming T., Yang JenPin, Hwang ReyChue, "A New Indoor Positioning Technique Based on Neural Network", Advanced Science Letters, Vol.19(5), July 2013 , pp. 2029-2033

[12] AL Nabhan, Mohammad Mousa, "Adaptive, reliable, and accurate positioning model for location-based services", Brunel University School of Engineering and Design PhD Theses, 2009

[13] Ray S., Blanco R., Goel, A.K., "Supporting Location-based Services in a Main-Memory Database", 15th IEEE International Conference on Mobile Data Management, 2014, pp.14-18

[14] Suprio Ray, Rolando Blanco, Anil K. Goel, "Enhanced database support for location-based services", Proceedings of the 4th ACM SIGSPATIAL International Workshop on GeoStreaming, 2013, p.22-25.

[15] Virrantaus, K., Markkula, J., Garmash, A., Terziyan, V., Veijalainen, J., Katanosov, A., Tirri, H., "Developing GIS-supported location-based services," Proceedings of the Second International Conference on Web Information Systems Engineering, 2001, vol.2, pp.66-75

[16] Jerry Gao, X. Bai, W. T. Tsai, and T. Uehara, "Mobile application testing: a tutorial", IEEE Computer, vol.47 (2), 2014, pp.26-35

[17] Ke Zhai, Bo Jiang, Chan, W.K., "Prioritizing Test Cases for Regression Testing of Location-Based Services: Metrics, Techniques, and Case Study," IEEE Transactions on Services Computing, 2014, vol.7, no.1, pp.54-67

[18] Hui-Chun Chua, Gwo-Jen Hwangb, Chin-Chung Tsaib, Judy C.R. Tsengc, "A two-tier test approach to developing location-aware mobile learning systems for natural science courses", Computers \& Education, vol.55(4), 2010, pp.1618-1627

[19] Bjornestad, S., Tessem, B., Nyre, L., "Design and Evaluation of a Location-Based Mobile News Reader," 4th IFIP International Conference on New Technologies, Mobility and Security (NTMS), 2011 pp.7-10

[20] Yu J, Tappenden A, Miller J et al., “A scalable testing framework for location-based services," journal of computer science and technology, vol.22(2),2009,386-44

[21] Oum-EI-Kheir Aktouf, Tao Zhang, Jerry Gao, Tadahiro Uehara, "Testing location-based function services for mobile applications", The First International Workshop on Mobile Cloud TaaS (MCTaaS 2015), 2015 , in press. 